\title{
Molecular Doping of the Hole-Transporting Layer for Efficient, Single-Step Deposited Colloidal Quantum Dot Photovoltaics
}

Ahmad R. Kirmani, F. Pelayo Garcia de Arquer, James Z. Fan, Jafar lqbal Khan, Grant Walters, Sjoerd Hoogland, Nimer Wehbe, Marcel M. Said, Stephen Barlow, Frédéric Laquai, Seth R. Marder, Edward H. Sargent, and Aram Amassian

ACS Energy Lett., Just Accepted Manuscript • DOI: 10.1021/acsenergylett.7b00540 • Publication Date (Web): 31 Jul 2017

Downloaded from http://pubs.acs.org on August 6, 2017

\section{Just Accepted}

"Just Accepted" manuscripts have been peer-reviewed and accepted for publication. They are posted online prior to technical editing, formatting for publication and author proofing. The American Chemical Society provides "Just Accepted" as a free service to the research community to expedite the dissemination of scientific material as soon as possible after acceptance. "Just Accepted" manuscripts appear in full in PDF format accompanied by an HTML abstract. "Just Accepted" manuscripts have been fully peer reviewed, but should not be considered the official version of record. They are accessible to all readers and citable by the Digital Object Identifier (DOI@). "Just Accepted" is an optional service offered to authors. Therefore, the "Just Accepted" Web site may not include all articles that will be published in the journal. After a manuscript is technically edited and formatted, it will be removed from the "Just Accepted" Web site and published as an ASAP article. Note that technical editing may introduce minor changes to the manuscript text and/or graphics which could affect content, and all legal disclaimers and ethical guidelines that apply to the journal pertain. ACS cannot be held responsible for errors or consequences arising from the use of information contained in these "Just Accepted" manuscripts. 


\title{
Molecular Doping of the Hole-Transporting Layer
}

\section{for Efficient, Single-Step Deposited Colloidal}

\section{Quantum Dot Photovoltaics}

\author{
Ahmad R. Kirmani ${ }^{1}$, F. Pelayo García de Arquer ${ }^{2}$, James Z. Fan ${ }^{2}$, Jafar I. Khan ${ }^{1}$, Grant \\ Walters $^{2}$, Sjoerd Hoogland ${ }^{2}$, Nimer Wehbe ${ }^{1}$, Marcel M. Said ${ }^{3}$, Stephen Barlow ${ }^{3}$, Frédéric \\ Laquai $^{1}$, Seth R. Marder ${ }^{3}$, Edward H. Sargent ${ }^{2}$, and Aram Amassian ${ }^{1, *}$ \\ ${ }^{1}$ King Abdullah University of Science and Technology (KAUST), KAUST Solar Center (KSC), \\ and, Physical Science and Engineering Division, Thuwal, 23955-6900, Saudi Arabia \\ ${ }^{2}$ Department of Electrical and Computer Engineering, University of Toronto, Toronto, Ontario \\ M5S 3G4, Canada. \\ ${ }^{3}$ School of Chemistry and Biochemistry and Center for Organic Photonics and Electronics, \\ Georgia Institute of Technology, 901 Atlantic Drive, Atlanta, GA, 30332-0400, United States
}

\section{AUTHOR INFORMATION \\ Corresponding Author \\ *E-mail: aram.amassian@kaust.edu.sa}


ABSTRACT: Employment of thin perovskite shells and metal halides as surface-passivants for colloidal quantum dots (CQDs) have been important, recent developments in CQD optoelectronics. These have opened the route to single-step deposited high-performing CQD solar cells. These promising architectures employ a CQD hole-transporting layer (HTL) whose intrinsically shallow Fermi level $\left(\mathrm{E}_{\mathrm{F}}\right)$ restricts band-bending at maximum power-point during solar cell operation limiting charge collection. Here, we demonstrate a generalized approach to effectively balance band-edge energy levels of the main CQD absorber and charge-transport layer for these high-performance solar cells. Briefly soaking the CQD HTL in a solution of the metalorganic p-dopant, molybdenum tris(1-(trifluoroacetyl)-2-(trifluoromethyl)ethane-1,2-dithiolene), effectively deepens its Fermi level, resulting in enhanced band bending at the HTL:absorber junction. This blocks the back-flow of photo-generated electrons, leading to enhanced photocurrent and fill factor compared to undoped devices. We demonstrate $9.0 \%$ perovskiteshelled and 9.5\% metal-halide-passivated CQD solar cells, both achieving $c a$. $10 \%$ relative enhancements over undoped baselines.

\section{TOC GRAPHICS}

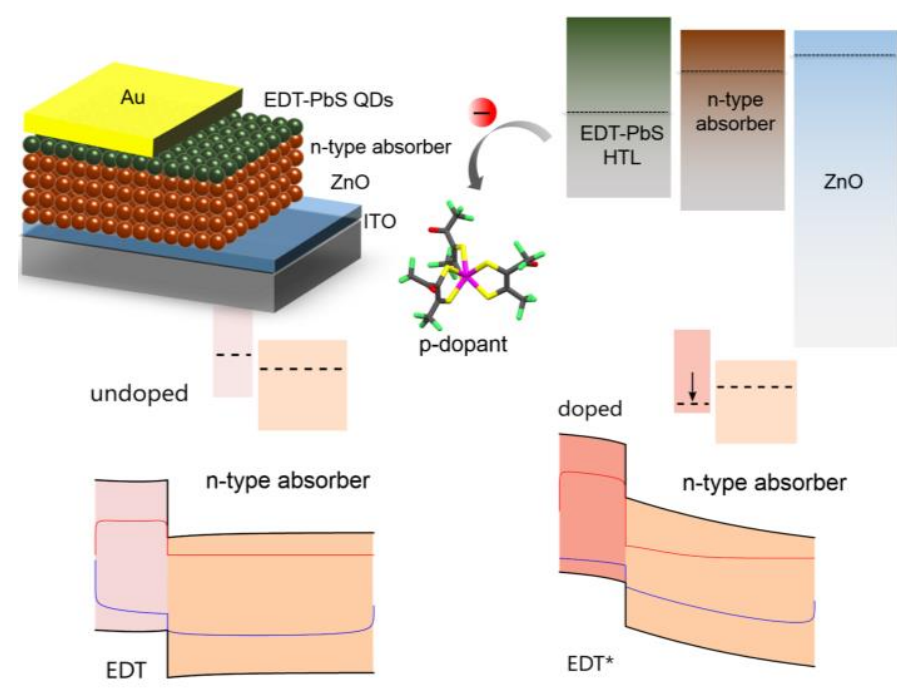


CQDs combine spectral tunability and narrow emission linewidths with solution-processability, making them strong prospects for applications in flexible displays, photovoltaics, and lasers. ${ }^{1-3}$ The last two years have witnessed significant inroads in surface chemistry and solar cell architectures, placing CQDs at the frontiers of the next generation optoelectronics. ${ }^{4-14}$

Among all reported strategies towards solution processed CQD solar cells, the halide-ligand passivation approach has been shown as the most efficient surface passivation scheme to date. ${ }^{6,15-}$ ${ }^{18}$ This has been paralleled by important developments in device engineering. ${ }^{7,}{ }^{10,19}$ A combination of these major breakthroughs has caused the certified power conversion efficiencies (PCEs) to leap beyond $11 \% .^{6-8,14}$

The standard procedure for the fabrication of CQD solar cells has until recently been the materials-wasteful and defect-introducing layer-by-layer (LbL) approach, which involves the sequential deposition of the insulating ligand-capped CQD layers followed by an aggressive solidstate ligand-exchange step and washing which cause incomplete passivation, undesirable pin-holes and cracks. ${ }^{20-21}$ Alternative approaches, such as solution-phase exchanges which aim at reducing the materials wastage and facilitating production, have recently been explored. ${ }^{21-22}$ These strategies, that allow single-step solar cell fabrication, have typically resulted in deteriorated electronic properties for the CQD solid, severely curtailing device performance. ${ }^{23-24}$ Recently, effective solution-phase exchange recipes relying on the use of methylammonium lead triiodide $\left(\mathrm{MAPbI}_{3}\right)$ perovskites and lead halide $\left(\mathrm{PbX}_{2}\right)$ ligands as capping shells for CQDs have been demonstrated. ${ }^{25-26}$ The resulting exchanged, stable CQDs inks ( $\mathrm{MAPbI}_{3}-\mathrm{PbS}$ and $\mathrm{PbX}$ - $\mathrm{PbS}$ ) have been used to fabricate efficient solar cells via single-step deposition, significantly streamlining active layer fabrication and curbing materials wastage. A thin layer of 1,2-ethanedithiol (EDT) ligand-capped PbS CQDs deposited on the active layer serves as the hole-transporting layer (HTL) 
and has become pervasive in recent reports of high performance CQD solar cells thanks to its ability to efficiently block the backflow of photo-generated electrons, thereby aiding efficient charge extraction by modifying the energetics at the interface. ${ }^{10}$ Normally, a ZnO nanoparticlebased thin film, directly deposited atop the transparent electrode via spin-casting, is employed as the electron-transporting metal-oxide layer (ETL) in these architectures. ${ }^{8,25-26}$

The halide-ligand passivated CQD solids are known to exhibit an n-type character, and their Fermi level $\left(\mathrm{E}_{\mathrm{F}}\right)$ is closer to the conduction band edge. ${ }^{10,15,27}$ This $\mathrm{n}$-character puts restrictions on the ETL, which needs to be highly degenerate in order for sufficient band bending to be retained at the maximum power point (MPP). ${ }^{8}$ Importantly, this also suggests that non-optimally doped EDT-PbS CQD solids would lead to similar issues with band bending near the hole-accepting electrode, affecting charge extraction at the MPP. Doping of the EDT-PbS CQD HTL has however remained largely unexplored thus far, with highly promising recent demonstrations by Loi and coworkers. $^{28-29}$

A controlled electron removal from the EDT-PbS CQD layer (p-doping) should effectively deepen its $\mathrm{E}_{\mathrm{F}}$. This would in turn result in a favorable band bending at the hole-accepting interface that improves charge extraction and, therefore, the solar cell performance. However, the introduction of p-dopants within the CQD solid can lead to harmful side effects, such as a significant decrease in carrier mobility, ${ }^{30-31}$ or a quenching of the exciton absorption. ${ }^{32}$ A strategy to controllably dope the CQD HTL without affecting its transport or absorption properties is therefore needed.

We took the view that this could be achieved by remote doping with small redox-active molecules. If appropriately designed, these molecules would be incorporated throughout the CQD 
HTL, withdrawing electrons without damaging transport characteristics. We designed a facile molecular p-doping recipe to selectively dope the EDT-PbS CQD based HTL. Such a recipe can be extended to a variety of CQD solar cell architectures which rely on a thin CQD solid as the HTL. $^{6-9,14,27,33}$ We employ a large electron affinity (EA) metal-organic complex, molybdenum tris(1-(trifluoroacetyl)-2-(trifluoromethyl)ethane-1,2-dithiolene), $\mathrm{Mo}\left(\mathrm{tfd}-\mathrm{COCF}_{3}\right) 3 .{ }^{34-35} \quad$ This molecule, as we have recently demonstrated, favors electron withdrawal in other CQD legacy absorber layers. ${ }^{36}$ Herein, we expand the application gamut of the molecular doping scheme to include more promising device architectures that make use of a CQD-based HTL and we specifically target the latter, rather than the active layer itself. We overcome the obvious technical challenge in these latest architectures of being able to selectively dope the HTL without altering the absorber layer. This approach is demonstrated to work for $\mathrm{MAPbI}_{3}-\mathrm{PbS}$ and $\mathrm{PbX} 2-\mathrm{PbS} \mathrm{CQD}$ absorbers, demonstrating its broad applicability to the latest generation of CQD ligand chemistries. The optimally p-doped HTL leads to an enhancement in the photocurrent and fill factor (FF) as compared to the undoped control solar cells, which ultimately leads to a ca. $10 \%$ increase in PCEs.

The perovskite-shelled CQD solar cell architecture is shown in Schematic 1a. Photon absorption and photocurrent generation take place primarily in the thick $\mathrm{MAPbI}_{3}-\mathrm{PbS} \mathrm{CQD}$ layer. ${ }^{10}$ Spin-cast $\mathrm{ZnO}$ nanoparticles were used as the electron-transporting layer (ETL). Schematic 1b shows a schematic of the Mo(tfd- $\left.\mathrm{COCF}_{3}\right)_{3}$ doping procedure. After the deposition of the EDT-PbS CQD hole extraction layer, the device is soaked briefly $(\sim 30 \mathrm{~s})$ in an acetonitrile (ACN) solution of $\mathrm{Mo}\left(\mathrm{tfd}-\mathrm{COCF}_{3}\right)_{3}$. ACN is known to be benign to the surface of CQDs unlike other protic solvents such as methanol $(\mathrm{MeOH}),{ }^{21}$ and is reported as an effective solvent for $\mathrm{Mo}\left(\mathrm{tfd}-\mathrm{COCF}_{3}\right)_{3}$ dopant delivery. ${ }^{36}$ We optimized the soaking time based on previous ACN-based ligand exchanges of 
oleic-acid capped CQDs in device architectures employing EDT-PbS CQD as the HTL, 4, 6, 8-10, 14, 25, 27 where $30-60$ s of soaking time leads to a complete ligand exchange. ${ }^{37}$

\section{(a)}

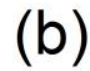

Schematic 1. (a) Solar cell architecture employing a $\mathrm{MAPbI}_{3}-\mathrm{PbS} \mathrm{CQD}$ absorber layer obtained via a single-step deposition. (b) Schematic depicting the idea behind the doping strategy. A deepLUMO metal-organic complex is used to extract electrons from the HTL, remotely p-doping the EDT layer. Black dashed lines represent the respective Fermi levels. The green arrow represents the expected electron transfer from the HTL to the dopant that results in p-doping (and not the flow of charges in the operating device).

To confirm that doping is predominantly limited to the CQD HTL overlayer, we analyzed the dopant concentration (Mo) through the CQD film using secondary ion mass spectrometry (SIMS). To achieve detection by SIMS, a $1 \mathrm{mg} \mathrm{mL}^{-1}$ dopant concentration was considered since dopant was hard to detect at lower concentrations due to poor signal:noise ratio in the spectra. The 3D distribution of Mo within the CQD solid reveals a strong confinement of the dopant to the top few tens of nanometers, with a concentration that is reduced by two orders of magnitude after $80 \mathrm{~nm}$ (Figure 1a). Figure 1b shows the corresponding SIMS depth profiles. F and Mo dopant species are found to decrease significantly beyond a few tens of nanometers. All the elements show a steep decrease beyond $\sim 150 \mathrm{~nm}$ which marks the bottom of the entire CQD layer considered for the SIMS measurements. It should be noted that these SIMS results represent only an approximation 
to the location of the dopant molecules. Being partially organic, the molecules are expected to be affected by preferential sputtering and matrix effects leading to quantification distortions. Thus, although Figure 1a confirms that the dopant molecules are predominantly situated in the EDTHTL, it is unclear whether a small amount of dopant may have also penetrated into the absorber layer. Nevertheless, we are encouraged by the predominantly near-surface confinement of dopant despite the use of a concentrated dopant solution (two orders of magnitude more concentrated than optimal solutions, as will be discussed below), which provides the stack with a larger supply of dopant for possible penetration into the active layer.

Successful doping of the CQDs was verified by photoluminescence (PL). EDT-PbS CQD films were fabricated on glass substrates for these measurements. Excitation was carried out using a 635 $\mathrm{nm}$ diode laser. The measurements confirm electron transfer from the CQDs to the dopant evident from the suppression of the PL intensity (Figure 1c). We also performed time-resolved PL (TRPL) on these samples to confirm improvement in charge extraction. p-doped HTLs are expected to demonstrate a reduced carrier lifetime suggestive of enhanced hole extraction, as seen, for example, in a recent report which demonstrated performance enhancement in perovskite solar cells by employing a p-doped HTL. ${ }^{38}$ The TRPL results are presented in Figure S10 and indicate a shorter lifetime for the doped sample. Fitting with mono-exponential decay functions indicates excited-state lifetimes of $\sim 202$ and $\sim 119$ ns for undoped and doped samples, respectively. No change in the intrinsic absorption is found as shown through the normalized transient-absorption characteristics (Figure 1d).

Efficient $\mathrm{p}$-doping is expected to lead to a deepening of the $\mathrm{E}_{\mathrm{F}}$ relative to the band positions and the vacuum level. We employed Kelvin Probe (KP) microscopy to characterize the work function difference of the different samples. KP measurements showed an increase in the work function of 
the EDT-PbS CQD solids as the doping concentration was increased (Figure S1). Changes to the overall work function, however, comprise of: i) changes to the $E_{F}$ and ii) vacuum level shifts originating from surface dipoles. We were interested in determining up to what extent p-doping affects the $\mathrm{E}_{\mathrm{F}}$ of the EDT-PbS CQD solids without creating a dipole moment. To have a deeper insight into the band structure changes, we resorted to ultraviolet photoemission spectroscopy (UPS) measurements. Extreme care should, however, be taken in interpreting UPS spectra of small bandgap CQDs, as pointed out by Miller et al. ${ }^{39}$ Figure 1e tracks the changes to the EDT-PbS CQD band structure for various doping concentrations. For these measurements, the films were immediately transferred into the ultra-high vacuum (UHV) chamber limiting the exposure to ambient environment. The conduction band minima (CBM) were approximated from the optical band gaps (Figure S2), an estimation which is justified for inorganic materials such as PbS CQDs given their small exciton binding energies. For low doping concentrations $\left(10^{-2}\right.$ and $10^{-3} \mathrm{mg} \mathrm{mL}^{-}$ ${ }^{1}$ ), the $\mathrm{E}_{\mathrm{F}}$ of the EDT-PbS CQDs is found to move closer to the valence band maxima (VBM), clearly suggestive of $\mathrm{p}$-doping. For larger doping concentrations, however, the $\mathrm{E}_{\mathrm{F}}$ is found to saturate well short of the band edge, without leading to degenerate doping of the CQDs. We speculate that this might occur due to a combination of the following factors: a) a fairly high number of trap states inside the band gap, and b) an electrostatic repulsion between the Mo(tfd$\left.\mathrm{COCF}_{3}\right)_{3}{ }^{-}$counterions, formed after the dopant molecules accept electrons effectively p-doping the CQDs. With increasing levels of doping, the anions form increasingly complete monolayers on the surface of the CQDs. Doping will then not only depend on the dopant-CQD energetics (EA of the dopant and VBM of the CQDs), but also on the electrostatic attraction between the positively charged CQDs and the negatively charged dopant anions, and the repulsion between the dopant anions. This remote doping scenario is different from the doping of organic semiconductors, and 
forces the anions to be close to one another leading to repulsive interactions limiting doping efficiency. Importantly, larger doping concentrations also involve a significant surface dipole formation leading to raising of the vacuum level $(\Delta \Phi)$. This has been discussed in detail by us in a previous report. ${ }^{36}$ These surface dipoles can potentially form if layers of unreacted dopant molecules start surrounding the CQD surfaces, a scenario which is likely for large doping concentrations. The secondary electron (SE) cutoffs and valence bands (VBs) for the various doping scenarios are shown in Figure S3. Importantly, the VB spectrum for the $1 \mathrm{mg} \mathrm{mL}^{-1}$ doping case represents significant deviations from that of undoped CQDs. This might be indicative of the presence of a monolayer of the dopant molecules atop the EDT-HTL for this particular doping concentration (although the SIMS data discussed indicates significant penetration of the dopant into the HTL), since UPS only detects the top $c a$. $1 \mathrm{~nm}$ of the surface. We, however, observe that the VB spectra for lower doping concentrations closely mimic that of PbS CQDs, ruling out any overlayer formation at lower concentrations (Figure $\mathrm{S} 3$ ). The $\Delta \mathrm{E}_{\mathrm{F}}$ measured from UPS agrees well with the magnitude of the shift of $\mathrm{Pb} 4 \mathrm{f}$ core levels to a lower binding energy, as observed in $\mathrm{X}$ ray photoelectron spectroscopy (XPS), shown in Figure S4. This behavior is similar to that previously observed when the same dopant was employed to dope absorber layers of MPA-capped PbS CQDs. ${ }^{36}$ 
(a)

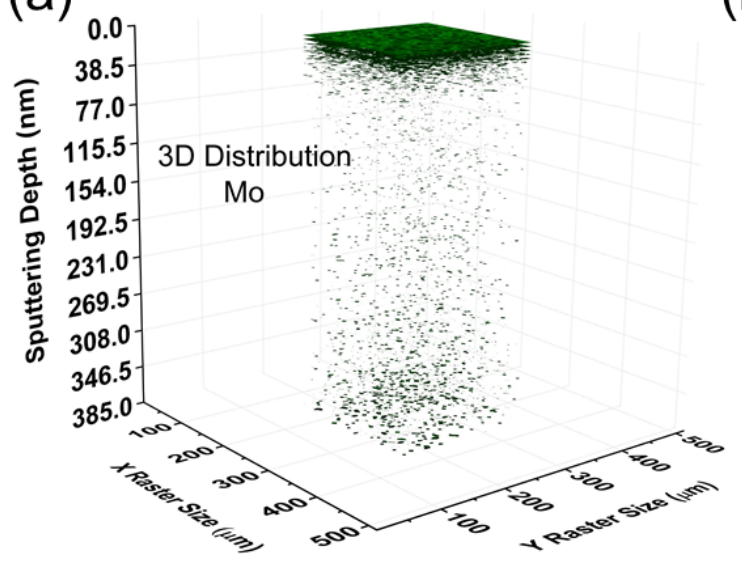

(c)

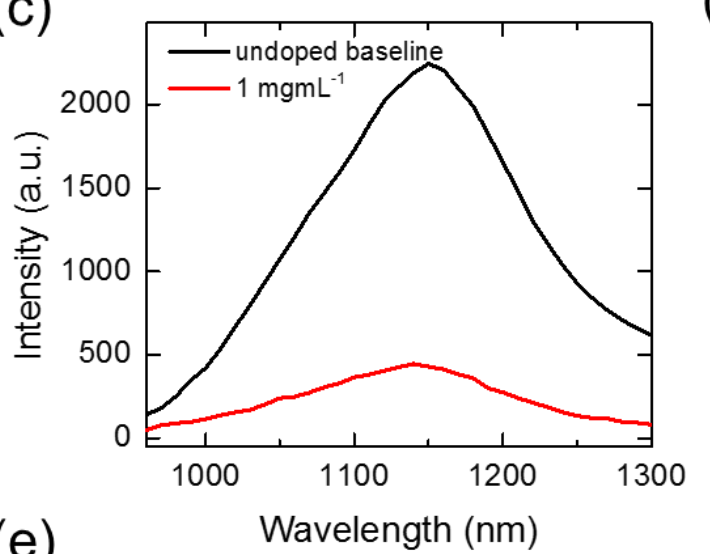

(b)

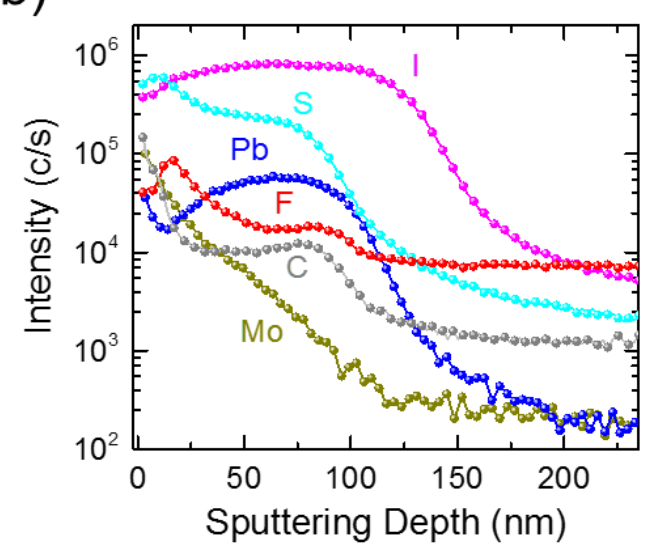

(d)

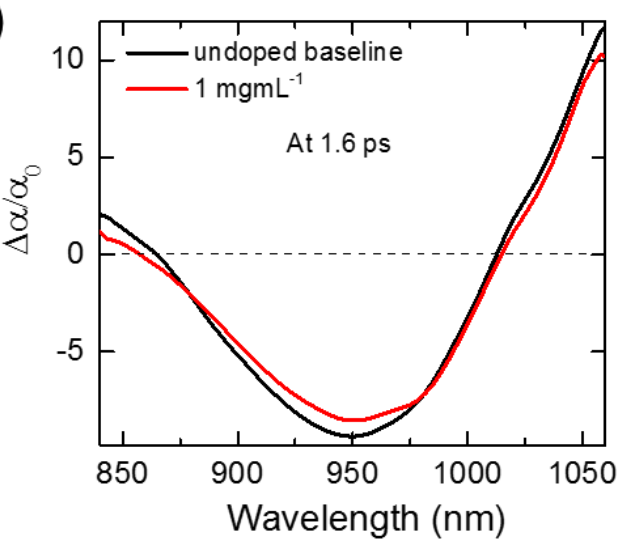

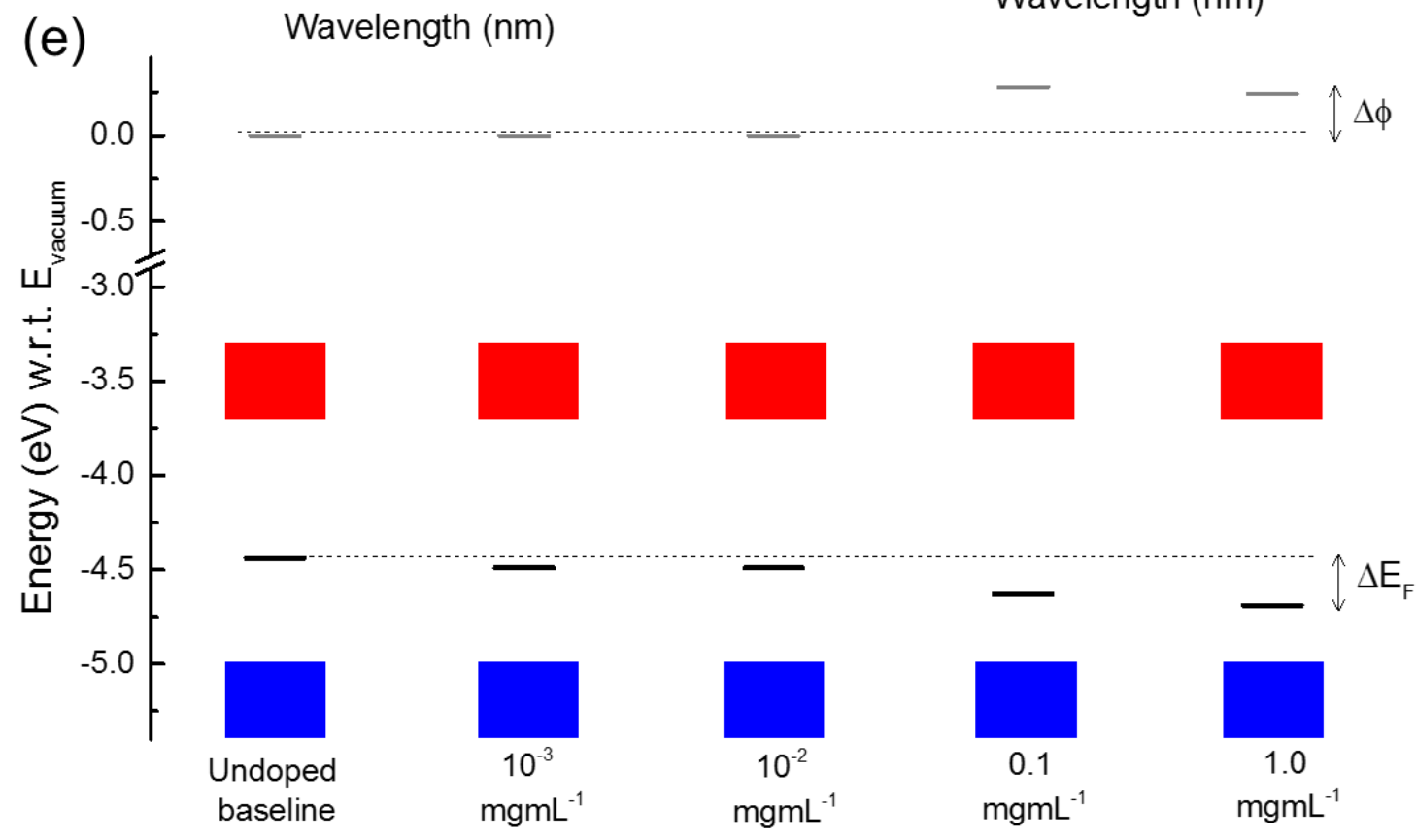

Figure 1. (a) 3D distribution for molybdenum from SIMS. (b) SIMS depth profiles for the various elements in the doped CQD HTL capping the CQD light absorber. (c) A PL suppression upon doping is suggestive of electron transfer from the CQD HTL to the dopant molecule. (d) Transientabsorption characteristics of the doped CQD solids remain unchanged relative to the undoped 
control. (e) Band structures of the EDT-PbS CQD solids under various doping scenarios as obtained from a combination of UPS and optical measurements. The dashed black line corresponding to $0 \mathrm{eV}$ represents the vacuum level for the undoped baseline. The gray dash for each band structure represents the corresponding vacuum level. The red and blue bands represent the conduction and valence bands of the CQD solids, respectively, while the black bars correspond to the $\mathrm{E}_{\mathrm{F}}$. The band structures have been put on a common scale according to the top of the VB, for clarity.

Encouraged by these findings, we made solar cells with doped EDT-PbS layers employing different dopant concentrations. The various steps involved in this facile molecular doping procedure are outlined in Figure 2. Figure $2 \mathrm{a}, 2 \mathrm{~b}$ and $2 \mathrm{c}$ show the various device parameters $\left(J_{S C}\right.$, $V_{O C}, \mathrm{FF}$ and PCE) as a function of the dopant concentration used for a large number of devices. Baseline undoped solar cells yield a PCE in the range of $8.1 \%$, which are on par with previously reported $\mathrm{MAPbI}_{3}$-shelled CQD solar cells. ${ }^{25}$ 

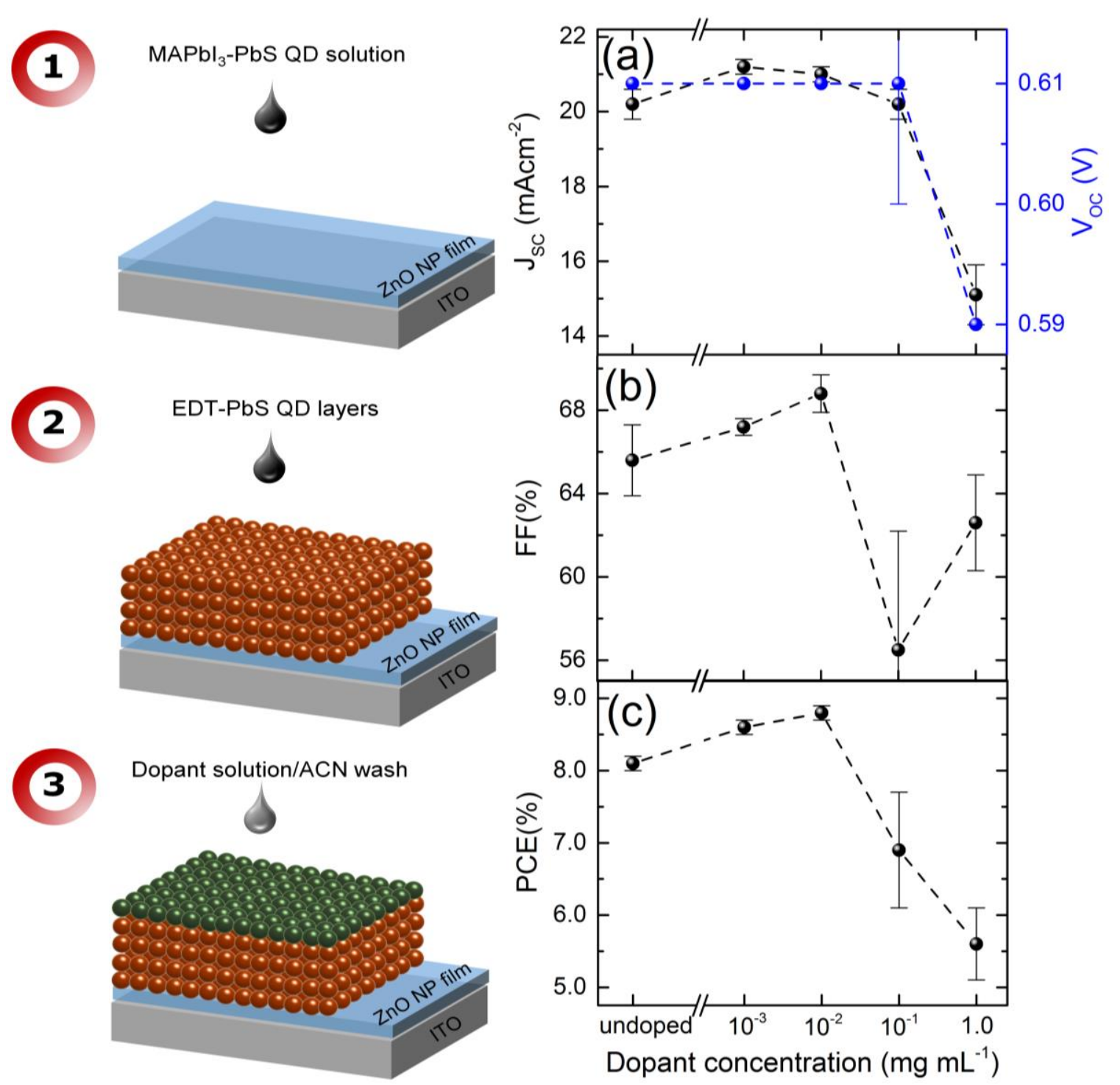

Figure 2. The various steps involved in the doping procedure are shown. The solar cell is fabricated in the standard way: (1) spin coating of the $\mathrm{MAPbI}_{3}-\mathrm{PbS}$ CQD ink on the ZnO-coated ITO glass, followed by (2) deposition of the EDT-PbS HTL. This assembly is then (3) soaked in the dopant solution (the desired concentration of $\mathrm{Mo}\left(\mathrm{tfd}-\mathrm{COCF}_{3}\right)_{3}$ in $\mathrm{ACN}$ ) for a 30 seconds followed by rinsing with ACN solvent to wash-off excess dopant molecules from the surface of the CQD solid. Device parameters $\left(J_{S C}, V_{O C}, \mathrm{FF}\right.$ and PCE) as a function of the dopant concentrations are shown in (a), (b) and (c). $10^{-2} \mathrm{mg} \mathrm{mL}^{-1}$ is found to be the optimized dopant concentration leading to the best performing solar cells. Error bars correspond to the standard deviation over a set of 6-12 devices.

Clear enhancements in $\mathrm{J}_{\mathrm{SC}}$ and FF are observed at low dopant concentrations $\left(10^{-3}\right.$ and $10^{-2} \mathrm{mg}$ $\mathrm{mL}^{-1}$ ); the PCE shows a maximum of $9.0 \%$ for the $10^{-2} \mathrm{mg} \mathrm{mL}^{-1}$ doped solar cell. The optimally p-doped HTL confers a smoother band alignment at the hole-collecting junction besides enhancing 
depletion in the absorber and results in improved charge generation and collection. Any gains in $V_{O C}$ are, however, compensated probably owing to the high density of surface trap states and increased scattering. ${ }^{28}$ The increase is statistically significant based on the increase seen in a number of devices. Increasing the dopant concentration further leads to a decrease of both $J_{\mathrm{SC}}$ and $\mathrm{FF}$, as well as a slight decrease in $V_{\mathrm{OC}}$ at the highest concentration, resulting in an overall performance degradation. A concentration of $10^{-2} \mathrm{mg} \mathrm{mL}^{-1}$ is therefore found to be the optimum dopant concentration, resulting in the most efficient charge extraction. We speculate that as the dopant concentration is increased above the optimum point $\left(10^{-2} \mathrm{mg} \mathrm{mL}^{-1}\right)$, the underlying $\mathrm{MAPbI}_{3}-\mathrm{PbS} \mathrm{CQD}$ photoactive layer might become affected, which in turn impairs device performance. We do see a small but non-negligible penetration of the dopant (Mo and F atoms) into the absorber layer for the $1 \mathrm{mg} \mathrm{mL}^{-1}$ doped film from SIMS (Figure 1(b)), as discussed above. UPS spectra provide clear clues into the impaired device performance at high doping concentrations. The UPS spectrum of the VB region for the $1 \mathrm{mg} \mathrm{mL}^{-1}$ case is suggestive of dopant segregation on the top of EDT-HTL (Figure S3). Higher doping concentrations can potentially result in a layer of unreacted dopant on CQD surfaces and act as barriers to hole flow. These high concentrations are associated with formation of large dipoles introducing important shifts in the CQD vacuum level. A severely shifted CQD vacuum level will disrupt charge flow near the Au electrode (the $\mathrm{Au}$ work function is $4.6 \mathrm{eV}$ ). Also, as has been shown recently in the case of graphene, higher doping concentrations utilizing similar dopants can lead to Coulomb scattering, adversely affecting charge transport. ${ }^{40}$

Table 1 summarizes the device data shown in Figure 2. The $J$ - $V$ curves for the best performing solar cells for each doping condition are shown in Figure 3a. The observed device performance enhancement is in good agreement with optoelectronic numerical predictions (Figure S5), which 
predict a FF and $\mathrm{J}_{\mathrm{SC}}$ increase as the doping concentration of the EDT layer approaches its optimum value. Importantly, the doped solar cells demonstrate photostability (Figure S6). The cells are found to show mild hysteresis, but no increase of the hysteretic behavior is observed upon doping (Figure S6). We postulate that the observed hysteresis might be linked to the use of perovskite shells.

Table 1. Device parameters $\left(J_{S C}, V_{O C}, \mathrm{FF}\right.$ and PCE) for the various doping concentrations. Statistics correspond to 6-12 devices. In brackets, the PCE of the best performing solar cells for each category, are shown.

\begin{tabular}{|c|c|c|c|c|}
\hline Device & $\mathrm{J}_{\mathrm{SC}}\left(\mathrm{mA} \mathrm{cm} \mathbf{c m}^{-2}\right)$ & $\mathbf{V}_{\text {OC }}(\mathbf{V})$ & FF $(\%)$ & PCE (\%) \\
\hline Undoped baseline & $20.2 \pm 0.4$ & $0.61 \pm 0.00$ & $65.6 \pm 1.7$ & $\begin{array}{c}8.1 \pm 0.1 \\
\quad(8.3)\end{array}$ \\
\hline $10^{-3} \mathrm{mg} \mathrm{mL}^{-1}$ & $21.2 \pm 0.2$ & $0.61 \pm 0.00$ & $67.2 \pm 0.4$ & $\begin{array}{c}8.6 \pm 0.1 \\
(8.8)\end{array}$ \\
\hline $10^{-2} \mathrm{mg} \mathrm{mL}^{-1}$ & $21.0 \pm 0.2$ & $0.61 \pm 0.00$ & $68.8 \pm 0.9$ & $\begin{array}{c}\mathbf{8 . 8} \pm \mathbf{0 . 1} \\
(9.0)\end{array}$ \\
\hline $10^{-1} \mathrm{mg} \mathrm{mL}^{-1}$ & $20.2 \pm 0.4$ & $0.61 \pm 0.01$ & $56.5 \pm 5.7$ & $\begin{array}{c}6.9 \pm 0.8 \\
(7.5)\end{array}$ \\
\hline $1.0 \mathrm{mg} \mathrm{mL}^{-1}$ & $15.1 \pm 0.8$ & $0.59 \pm 0.00$ & $62.6 \pm 2.3$ & $\begin{array}{c}5.6 \pm 0.5 \\
(6.1)\end{array}$ \\
\hline
\end{tabular}

The observed enhancement in device performance for solar cells using a p-doped EDT-PbS HTL agrees well with the increase in external quantum efficiency (EQE) under short-circuit current conditions (Figure S7). The EQE improvement for the optimally-doped solar cell, especially at the near infrared region, is consistent with the improvement in charge extraction leading to an increase in $\mathrm{FF}$ and $\mathrm{J}_{\mathrm{SC}}$. In order to assess the improvement attained by increased band bending at the maximum power point, we compare the EQE of undoped and optimally doped solar cells at these operating conditions (Figure 3b). The EQE is found to increase over the entire wavelength 
spectrum, but the enhancement is more significant in the near infrared ( $>10 \%$ at the exciton peak). This correlates well with the device performance increase attributed to a stronger p-type character of the EDT-PbS layer, as these photocharges are generated closer to this interface. Fabry-Perot interference results in a decrease of EQE around $800 \mathrm{~nm}$ in both cases.

The impact of doping on the interfacial band structure at the HTL:absorber interface is schematized in Figures $3 \mathrm{c}$ and $3 \mathrm{~d}$. For the case of a standard, undoped HTL (Figure 3c), the extraction of charges that are photogenerated close to the back interface face an unfavorable energy barrier in the band structure largely leading to carrier recombination. The doped HTL, which we modelled as $N_{\text {acceptor }} \sim 10^{18} \mathrm{~cm}^{-3}$ (based on previous report) ${ }^{41}$ to better illustrate the mechanism, on the other hand, leads to removal of this 'kink' and causes a favorable bending at the interface (Figure 3d). This finding is in good agreement with the observed increase in EQE at the exciton peak (Figure 3b), since these are the photons that are absorbed closer to the EDT back-interface. 
(a)
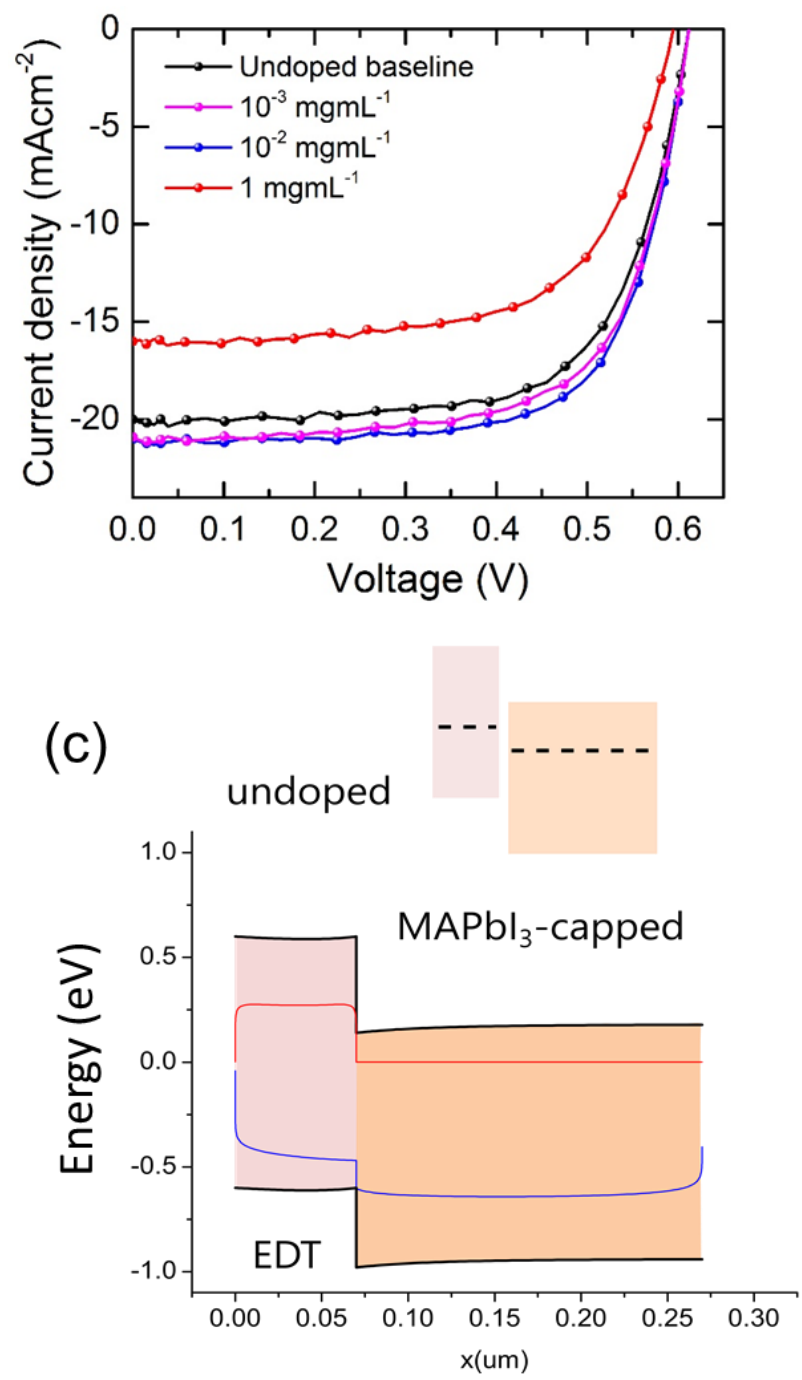

(b)
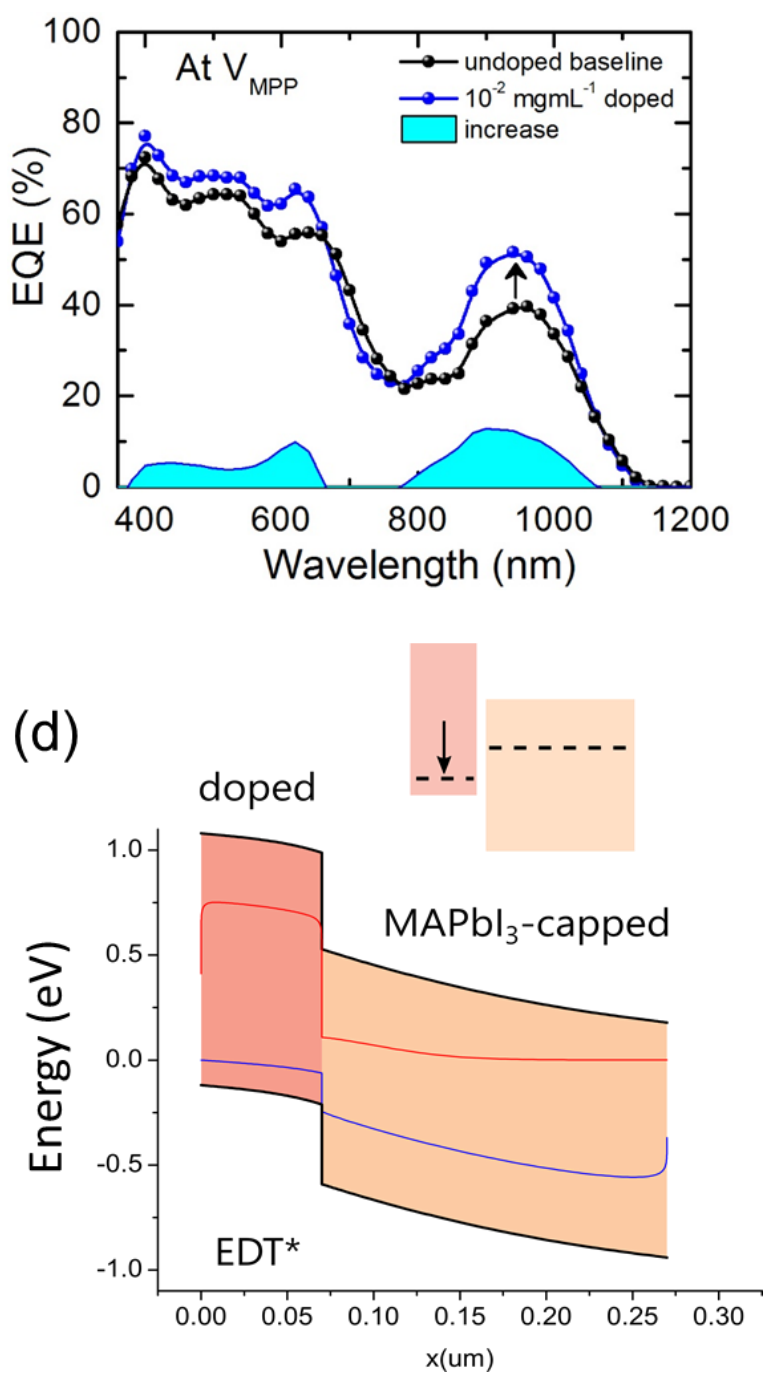

Figure 3. (a) $J-V$ curves for the various HTL doping scenarios are shown. Low dopant concentrations $\left(10^{-3}, 10^{-2} \mathrm{mg} \mathrm{mL}^{-1}\right)$ improve device performance, whereas a high concentration (1.0 $\mathrm{mg} \mathrm{mL}^{-1}$ ) leads to severe degradation. (b) EQE spectra for the undoped and optimally doped $\left(10^{-2} \mathrm{mg} \mathrm{mL}^{-1}\right)$ solar cells taken at $\mathrm{V}_{\mathrm{MPP}}(\sim 0.5 \mathrm{~V})$ conditions. Optimal doping (blue) leads an increase in charge collection compared to the undoped baseline (black) which is especially evident in the near infrared region. This illustrates the beneficial effect of an increased band banding in the photoactive layer at the HTL interface. (c-d) Schematics show that an undoped HTL causes an unfavorable 'kink' in the band structure at the HTL:absorber interface which is removed upon optimal doping of the HTL. The insets show the scenarios before thermodynamic equilibrium (black dashes represent the Fermi level positions). Charge collection efficiency increases for the optimally doped case.

To investigate whether the molecular doping scheme for the CQD HTL demonstrated for $\mathrm{MAPbI}_{3}-\mathrm{PbS}$ cells is general in application, we turned our attention to the recently reported $\mathrm{PbX}_{2}-$ 
PbS CQD based solar cells. ${ }^{26}$ These CQDs benefit from a hard nanocrystal surface owing to an increased halide surface coverage, effectively suppressing low-energy phonon modes prone to coupling with electronic transitions and creating carrier traps. ${ }^{26,42}$ The device architecture is shown in Figure 4a and differs from the $\mathrm{MAPbI}_{3}-\mathrm{PbS}$ CQD devices in employing a $\mathrm{PbX}_{2}-\mathrm{PbS} \mathrm{CQD}$ absorber layer. Nevertheless, it employs an EDT-PbS CQD HTL for efficient carrier extraction similar to the $\mathrm{MAPbI}_{3}-\mathrm{PbS} \mathrm{CQD}$ cells investigated above. Doping the CQD HTL with the molecular doping conditions optimized above for the case of $\mathrm{MAPbI}_{3}-\mathrm{PbS}$ devices, we observed similar device performance increase (Figure $4 \mathrm{~b}$ ). Enhancement in $\mathrm{J}_{\mathrm{SC}}$ and FF (Table S1) led to a statistically reproducible $c a$. $10 \%$ relative increase in PCEs compared to undoped control (black curve; PCE $=8.5 \%$ ) and resulted in $9.5 \%$ efficient solar cells (blue curve). The doped cells are found to demonstrate acceptable levels of photostability (Figure S8). A histogram representing device statistics (Figure 4c) confirms reproducibility of our PCE enhancements. Importantly, we find that the doped devices remain within $95 \%$ of their initial PCEs even after 2 months of storage in ambient environment, closely matching the stability of the undoped control cells (Figure S9). Although the air-stability of these molecular dopants is well-known, ${ }^{34-35,43}$ our findings demonstrate the reliability of optoelectronic devices that employ these dopants. 

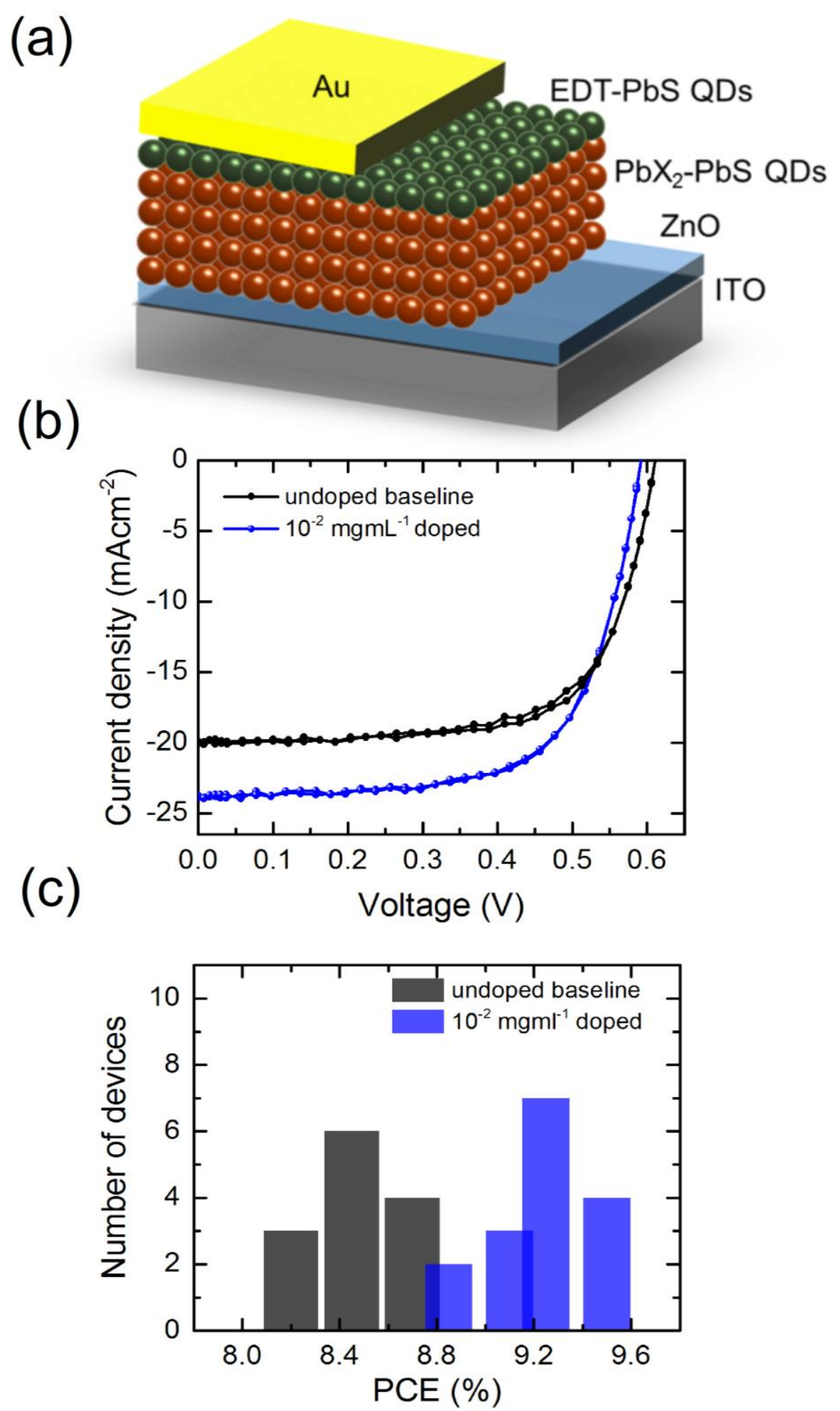

Figure 4. (a) Solar cell architecture employing a $\mathrm{PbX}_{2}-\mathrm{PbS} \mathrm{CQD}$ absorber layer obtained via a single-step deposition. (b) $J-V$ curves for the baseline (black curve) using an undoped EDT-PbS CQD HTL and a $10^{-2} \mathrm{mg} \mathrm{mL}^{-1}$ doped CQD HTL (blue curve). (c) Histogram showing device statistics for the undoped and doped cases. 
In summary, we have demonstrated that charge-carrier extraction in the latest brand of high performing, single-step deposited PbS CQD based solar cells is limited by the moderate p-type character of the EDT-PbS CQD HTL, which allows only limited band bending under maximum power point operation. We address this problem by introducing a molecular dopant which consists of a large-EA metal-organic complex $\mathrm{Mo}\left(\mathrm{tfd}-\mathrm{COCF}_{3}\right)_{3}$. When incorporated into the EDT-PbS layer, these molecules withdraw excess electrons, conferring a stronger p-type doping to the HTL. This ultimately results in an increased built-in field at this interface that is sustained even at maximum power point conditions, facilitating charge separation and collection. As a consequence, a $c$. $10 \%$ PCE increase is obtained compared to control samples. Our HTL p-doping recipe involving metal-organic complexes is applicable, in general, to the high-performing CQD solar cell architectures which rely on CQD HTLs for efficient charge collection.

\section{AUTHOR INFORMATION}

\section{Corresponding Author}

*E-mail: aram.amassian@kaust.edu.sa

\section{Author Contributions}

The manuscript was written through contributions of all authors. All authors have given approval to the final version of the manuscript.

\section{ACKNOWLEDGEMENTS}

The authors thank Yadong Zhang (Georgia Institute of Technology) for the chemical synthesis of the metal-organic complex, Mo(tfd-COCF 3$)_{3}$, used in this study. F.P.G.A., J. Z. F., G. W., S. H., and E. H. S. thank the Award KUS-11-009-21 from King Abdullah University of Science and Technology (KAUST), the Ontario Research Fund - Research Excellence Program, and the Natural Sciences and Engineering Research Council of Canada (NSERC). M. M. S., S.B., and S. 
R. M. thank the Office of Naval Research for support through (N00014-14-1-0126). F.P.G.A. acknowledges financial support from the Connaught Fund.

\section{ASSOCIATED CONTENT}

Supporting Information. Details on the experimental methods, KP data, steady-state absorption spectra, UPS and XPS measurements, data on the material parameters used for optoelectronic simulations (SCAPS) and simulated device parameters are included in the Supporting Information.

\section{REFERENCES}

(1) Yuan, M.; Liu, M.; Sargent, E. H. Colloidal Quantum Dot Solids for Solution-Processed Solar Cells. Nat. Energy 2016, 1, 16016.

(2) Gong, X.; Yang, Z.; Walters, G.; Comin, R.; Ning, Z.; Beauregard, E.; Adinolfi, V.; Voznyy, O.; Sargent, E. H. Highly Efficient Quantum Dot Near-Infrared Light-Emitting Diodes. Nat. Photon. 2016, 10, 253-257.

(3) Adachi, M. M.; Fan, F.; Sellan, D. P.; Hoogland, S.; Voznyy, O.; Houtepen, A. J.; Parrish, K. D.; Kanjanaboos, P.; Malen, J. A.; Sargent, E. H. Microsecond-Sustained Lasing from Colloidal Quantum Dot Solids. Nat. Commun. 2015, 6, 8694.

(4) Cao, Y.; Stavrinadis, A.; Lasanta, T.; So, D.; Konstantatos, G. The Role of Surface Passivation for Efficient and Photostable PbS Quantum Dot Solar Cells. Nat. Energy 2016, 1,16035 .

(5) Kagan, C. R.; Lifshitz, E.; Sargent, E. H.; Talapin, D. V. Building Devices from Colloidal Quantum Dots. Science 2016, 353, aac5523.

(6) Lan, X.; Voznyy, O.; García de Arquer, F. P.; Liu, M.; Xu, J.; Proppe, A. H.; Walters, G.; Fan, F.; Tan, H.; Liu, M.; et al. 10.6\% Certified Colloidal Quantum Dot Solar Cells Via Solvent-Polarity-Engineered Halide Passivation. Nano Lett. 2016, 16, 4630-4634.

(7) Lan, X.; Voznyy, O.; Kiani, A.; García de Arquer, F. P.; Abbas, A. S.; Kim, G.-H.; Liu, M.; Yang, Z.; Walters, G.; Xu, J.; et al. Passivation Using Molecular Halides Increases Quantum Dot Solar Cell Performance. Adv. Mater. 2016, 28, 299-304.

(8) Liu, M.; de Arquer, F. P. G.; Li, Y.; Lan, X.; Kim, G.-H.; Voznyy, O.; Jagadamma, L. K.; Abbas, A. S.; Hoogland, S.; Lu, Z.; et al. Double-Sided Junctions Enable High-Performance Colloidal-Quantum-Dot Photovoltaics. Adv. Mater. 2016, 28, 4142-4148.

(9) Kim, G.-H.; García de Arquer, F. P.; Yoon, Y. J.; Lan, X.; Liu, M.; Voznyy, O.; Yang, Z.; Fan, F.; Ip, A. H.; Kanjanaboos, P.; et al. High-Efficiency Colloidal Quantum Dot Photovoltaics via Robust Self-Assembled Monolayers. Nano Lett. 2015, 15, 7691-7696.

(10) Chuang, C.-H. M.; Brown, P. R.; Bulović, V.; Bawendi, M. G. Improved Performance and Stability in Quantum dot Solar Cells through Band Alignment engineering. Nat. Mater. 2014, 13, 796-801. 
(11) Kim, B.-S.; Neo, D. C. J.; Hou, B.; Park, J. B.; Cho, Y.; Zhang, N.; Hong, J.; Pak, S.; Lee, S.; Sohn, J. I.; et al. High Performance PbS Quantum Dot/Graphene Hybrid Solar Cell with Efficient Charge Extraction. ACS App. Mater. Inter. 2016, 8, 13902-13908.

(12) Neo, D. C. J.; Zhang, N.; Tazawa, Y.; Jiang, H.; Hughes, G. M.; Grovenor, C. R. M.; Assender, H. E.; Watt, A. A. R. Poly(3-Hexylthiophene-2,5-Diyl) as a Hole Transport Layer for Colloidal Quantum Dot Solar Cells. ACS App. Mater. Inter. 2016, 8, 12101-12108.

(13) Zhang, N.; Neo, D. C. J.; Tazawa, Y.; Li, X.; Assender, H. E.; Compton, R. G.; Watt, A. A. R. Narrow Band Gap Lead Sulfide Hole Transport Layers for Quantum Dot Photovoltaics. ACS App. Mater. Inter. 2016, 8, 21417-21422.

(14) Jin, Z.; Yuan, M.; Li, H.; Yang, H.; Zhou, Q.; Liu, H.; Lan, X.; Liu, M.; Wang, J.; Sargent, E. H.; et al. Graphdiyne: An Efficient Hole Transporter for Stable High-Performance Colloidal Quantum Dot Solar Cells. Adv. Funct. Mater. 2016, 26, 5284-5289.

(15) Ning, Z.; Voznyy, O.; Pan, J.; Hoogland, S.; Adinolfi, V.; Xu, J.; Li, M.; Kirmani, A. R.; Sun, J.-P.; Minor, J.; et al. Air-Stable n-Type Colloidal Quantum Dot Solids. Nat. Mater. 2014, 13, 822-828.

(16) Ning, Z.; Ren, Y.; Hoogland, S.; Voznyy, O.; Levina, L.; Stadler, P.; Lan, X.; Zhitomirsky, D.; Sargent, E. H. All-Inorganic Colloidal Quantum Dot Photovoltaics Employing SolutionPhase Halide Passivation. Adv. Mater. 2012, 24, 6295-6299.

(17) Ip, A. H.; Kiani, A.; Kramer, I. J.; Voznyy, O.; Movahed, H. F.; Levina, L.; Adachi, M. M.; Hoogland, S.; Sargent, E. H. Infrared Colloidal Quantum Dot Photovoltaics via Coupling Enhancement and Agglomeration Suppression. ACS Nano 2015, 9, 8833-8842.

(18) Wang, R.; Shang, Y.; Kanjanaboos, P.; Zhou, W.; Ning, Z.; Sargent, E. H. Colloidal Quantum Dot Ligand Engineering for High Performance Solar Cells. Energy Env. Sci. 2016, 9, 1130-1143.

(19) Maraghechi, P.; Labelle, A. J.; Kirmani, A. R.; Lan, X.; Adachi, M. M.; Thon, S. M.; Hoogland, S.; Lee, A.; Ning, Z.; Fischer, A.; et al. The Donor-Supply Electrode Enhances Performance in Colloidal Quantum Dot Solar Cells. ACS Nano 2013, 7, 6111-6116.

(20) Carey, G. H.; Chou, K. W.; Yan, B.; Kirmani, A. R.; Amassian, A.; Sargent, E. H. Materials Processing Strategies for Colloidal Quantum Dot Solar Cells: Advances, Present-Day Limitations, and Pathways to Improvement. MRS Commun. 2013, 3, 83-90.

(21) Kirmani, A. R.; Carey, G. H.; Abdelsamie, M.; Yan, B.; Cha, D.; Rollny, L. R.; Cui, X.; Sargent, E. H.; Amassian, A. Effect of Solvent Environment on Colloidal-Quantum-Dot Solar-Cell Manufacturability and Performance. Adv. Mater. 2014, 26, 4717-4723.

(22) Giansante, C.; Carbone, L.; Giannini, C.; Altamura, D.; Ameer, Z.; Maruccio, G.; Loiudice, A.; Belviso, M. R.; Cozzoli, P. D.; Rizzo, A.; et al. Colloidal Arenethiolate-Capped Pbs Quantum Dots: Optoelectronic Properties, Self-Assembly, and Application in Solution-Cast Photovoltaics. J. Phys. Chem. C 2013, 117, 13305-13317.

(23) Fischer, A.; Rollny, L.; Pan, J.; Carey, G. H.; Thon, S. M.; Hoogland, S.; Voznyy, O.; Zhitomirsky, D.; Kim, J. Y.; Bakr, O. M.; et al. Directly Deposited Quantum Dot Solids Using a Colloidally Stable Nanoparticle Ink. Adv. Mater. 2013, 25, 5742-5749.

(24) Ning, Z.; Dong, H.; Zhang, Q.; Voznyy, O.; Sargent, E. H. Solar Cells Based on Inks of nType Colloidal Quantum Dots. ACS Nano 2014, 8, 10321-10327.

(25) Yang, Z.; Janmohamed, A.; Lan, X.; García de Arquer, F. P.; Voznyy, O.; Yassitepe, E.; Kim, G.-H.; Ning, Z.; Gong, X.; Comin, R.; et al. Colloidal Quantum Dot Photovoltaics Enhanced by Perovskite Shelling. Nano Lett. 2015, 15, 7539-7543. 
(26) Liu, M.; Voznyy, O.; Sabatini, R.; Garcia de Arquer, F. P.; Munir, R.; Balawi, A. H.; Lan, X.; Fan, F.; Walters, G.; Kirmani, A. R.; et al. Hybrid Organic-Inorganic Inks Flatten the Energy Landscape in Colloidal Quantum Dot Solids. Nat. Mater. 2017, 16, 258-263.

(27) Brown, P. R.; Kim, D.; Lunt, R. R.; Zhao, N.; Bawendi, M. G.; Grossman, J. C.; Bulović, V. Energy Level Modification in Lead Sulfide Quantum Dot Thin Films through Ligand Exchange. ACS Nano 2014, 8, 5863-5872.

(28) Speirs, M. J.; Balazs, D. M.; Dirin, D. N.; Kovalenko, M. V.; Loi, M. A. Increased Efficiency in pn-Junction PbS QD Solar Cells Via NaHS Treatment of the p-Type Layer. Appl. Phys. Lett. 2017, 110, 103904.

(29) Speirs, M. J.; Dirin, D. N.; Abdu-Aguye, M.; Balazs, D. M.; Kovalenko, M. V.; Loi, M. A. Temperature Dependent Behaviour of Lead Sulfide Quantum Dot Solar Cells and Films. Energy Env. Sci. 2016, 9, 2916-2924.

(30) Baltazar, J.; Sojoudi, H.; Paniagua, S. A.; Zhang, S.; Lawson, R. A.; Marder, S. R.; Graham, S.; Tolbert, L. M.; Henderson, C. L. Photochemical Doping and Tuning of the Work Function and Dirac Point in Graphene Using Photoacid and Photobase Generators. Adv. Funct. Mater. 2014, 24, 5147-5156.

(31) Engel, J. H.; Surendranath, Y.; Alivisatos, A. P. Controlled Chemical Doping of Semiconductor Nanocrystals Using Redox Buffers. J. Am. Chem. Soc. 2012, 134, 1320013203.

(32) Koh, W.-k.; Koposov, A. Y.; Stewart, J. T.; Pal, B. N.; Robel, I.; Pietryga, J. M.; Klimov, V. I. Heavily Doped n-Type PbSe and PbS Nanocrystals Using Ground-State Charge Transfer from Cobaltocene. Sci. Rep. 2013, 3, 2004.

(33) Azmi, R.; Oh, S.-H.; Jang, S.-Y. High-Efficiency Colloidal Quantum Dot Photovoltaic Devices Using Chemically Modified Heterojunctions. ACS Energy Lett. 2016, 1, 100-106.

(34) Paniagua, S. A.; Baltazar, J.; Sojoudi, H.; Mohapatra, S. K.; Zhang, S.; Henderson, C. L.; Graham, S.; Barlow, S.; Marder, S. R. Production of Heavily n- and p-Doped CVD Graphene with Solution-Processed Redox-Active Metal-Organic Species. Mater. Horiz. 2014, 1, 111 115.

(35) Mohapatra, S. K.; Zhang, Y.; Sandhu, B.; Fonari, M. S.; Timofeeva, T. V.; Marder, S. R.; Barlow, S. Synthesis, Characterization, and Crystal Structures of Molybdenum Complexes of Unsymmetrical Electron-Poor Dithiolene Ligands. Polyhedron 2016, 116, 88-95.

(36) Kirmani, A. R.; Kiani, A.; Said, M. M.; Voznyy, O.; Wehbe, N.; Walters, G.; Barlow, S.; Sargent, E. H.; Marder, S. R.; Amassian, A. Remote Molecular Doping of Colloidal Quantum Dot Photovoltaics. ACS Energy Lett. 2016, 1, 922-930.

(37) Kim, B.-S.; Hong, J.; Hou, B.; Cho, Y.; Sohn, J. I.; Cha, S.; Kim, J. M. Inorganic-Ligand Exchanging Time Effect in PbS Quantum Dot Solar Cell. Appl. Phys. Lett. 2016, 109, 063901.

(38) Liu, D.; Li, Y.; Yuan, J.; Hong, Q.; Shi, G.; Yuan, D.; Wei, J.; Huang, C.; Tang, J.; Fung, M.-K. Improved Performance of Inverted Planar Perovskite Solar Cells with F4-TCNQ Doped PEDOT:PSS Hole Transport Layers. J. Mater. Chem. A 2017.

(39) Miller, E. M.; Kroupa, D. M.; Zhang, J.; Schulz, P.; Marshall, A. R.; Kahn, A.; Lany, S.; Luther, J. M.; Beard, M. C.; Perkins, C. L.; et al. Revisiting the Valence and Conduction Band Size Dependence of PbS Quantum Dot Thin Films. ACS Nano 2016, 10, 3302-3311.

(40) Mansour, A. E.; Said, M. M.; Dey, S.; Hu, H.; Zhang, S.; Munir, R.; Zhang, Y.; Moudgil, K.; Barlow, S.; Marder, S. R.; et al. Facile Doping and Work-Function Modification of Few- 
Layer Graphene Using Molecular Oxidants and Reductants. Adv. Funct. Mater. 2017, 27, 1602004.

(41) Zhitomirsky, D.; Voznyy, O.; Levina, L.; Hoogland, S.; Kemp, K. W.; Ip, A. H.; Thon, S. M.; Sargent, E. H. Engineering Colloidal Quantum Dot Solids within and Beyond the Mobility-Invariant Regime. Nat. Commun. 2014, 5, 3803.

(42) Bozyigit, D.; Yazdani, N.; Yarema, M.; Yarema, O.; Lin, W. M. M.; Volk, S.; Vuttivorakulchai, K.; Luisier, M.; Juranyi, F.; Wood, V. Soft Surfaces of Nanomaterials Enable Strong Phonon Interactions. Nature 2016, 531, 618-622.

(43) Tarasov, A.; Zhang, S.; Tsai, M.-Y.; Campbell, P. M.; Graham, S.; Barlow, S.; Marder, S. R.; Vogel, E. M. Controlled Doping of Large-Area Trilayer $\mathrm{MoS}_{2}$ with Molecular Reductants and Oxidants. Adv. Mater. 2015, 27, 1175-1181. 